\title{
ON THE HILBERT GEOMETRY OF PRODUCTS
}

\author{
CONSTANTIN VERNICOS
}

(Communicated by Michael Wolf)

\begin{abstract}
We prove that the Hilbert geometry of a product of convex sets is bi-lipschitz equivalent to the direct product of their respective Hilbert geometries. We also prove that the volume entropy is additive with respect to product and that amenability of a product is equivalent to the amenability of each term.
\end{abstract}

\section{INTRODUCTION AND STATEMENT OF RESULTS}

Hilbert geometries are simple metric geometries defined in the interior of an open convex set thanks to cross-ratios. They are generalisations of the projective model of hyperbolic geometry. Because of their definition they are invariant by the action of projective transformations. Among all these geometries, those admitting a discrete subgroup of their isometries acting co-compactly, commonly known as divisible Hilbert geometries or divisible convex sets, play an important part. For instance we can find examples of such geometries, which are hyperbolic in the sense of Gromov with a quotient which does not admit any Riemannian hyperbolic metric Ben06.

The present paper focuses on the product of Hilbert geometries, and takes its roots in the following question: Does the product of two divisible convex sets give a divisible convex set? The answer to that question is no and is given by a very simple example, the Hilbert geometry of the square. Indeed, the Hilbert geometry of the segment $[-1,1]$, which is isometric to the real line, is divisible. However, the product of two such segments, which is a square in $\mathbb{R}^{2}$, endowed with its Hilbert geometry, is not a divisible convex set, which is related to the fact that one can't immerse $P G L(2, \mathbb{R}) \times P G L(2, \mathbb{R})$ into $P G L(3, \mathbb{R})$.

However, following B. Colbois, C. Vernicos, and P. Verovic [CV11] the Hilbert geometry of a polygon is bi-lipschitz equivalent to $\mathbb{R}^{2}$. In the light of that example we asked ourselves what is the relation between the Hilbert geometry of a product and the product of Hilbert geometries.

Before stating our results let us first present a more precise definition of a what a Hilbert geometry is and introduce the invariants involved in the present work.

A proper open set in $\mathbb{R}^{n}$ is a set not containing a whole line. A Hilbert geometry $\left(\mathcal{C}, d_{\mathcal{C}}\right)$ is a non-empty proper open convex set $\mathcal{C}$ on $\mathbb{R}^{n}$ (that we shall call convex domain) with the Hilbert distance $d_{\mathcal{C}}$ defined as follows: for any distinct points $p$ and $q$ in $\mathcal{C}$, the line passing through $p$ and $q$ meets the boundary $\partial \mathcal{C}$ of $\mathcal{C}$ at two points $a$ and $b$, such that one walking on the line goes consecutively by $a, p, q, b$.

Received by the editors January 30, 2012 and, in revised form, February 17, 2014.

2010 Mathematics Subject Classification. Primary 53C60; Secondary 53C24, 58B20, 53A20. 
Then we define

$$
d_{\mathcal{C}}(p, q)=\frac{1}{2} \ln [a, p, q, b]
$$

where $[a, p, q, b]$ is the cross-ratio of $(a, p, q, b)$, i.e.,

$$
[a, p, q, b]=\frac{\|q-a\|}{\|p-a\|} \times \frac{\|p-b\|}{\|q-b\|}>1
$$

with $\|\cdot\|$ the canonical euclidean norm in $\mathbb{R}^{n}$. If either $a$ or $b$ is at infinity the corresponding ratio will be taken equal to 1 .

Note that the invariance of the cross-ratio by a projective map implies the invariance of $d_{\mathcal{C}}$ by such a map.

The Hilbert distance $d_{\mathcal{C}}$ is the length distance associated to a $C^{0}$ Finsler metric $F_{\mathcal{C}}$ which can be descibed as follows: if $p \in \mathcal{C}$, then $F_{\mathcal{C}}(p, \cdot)$ is the support function of the Minkowski sum

$$
1 / 2\left(\mathcal{C}_{p}^{*}-\mathcal{C}_{p}^{*}\right)=\left\{\frac{1}{2} x-\frac{1}{2} y \mid x, y \in \mathcal{C}_{p}^{*}\right\}
$$

where $\mathcal{C}_{p}^{*}$ is the dual convex set associated to $\mathcal{C}$ when $p$ is taken as the origin.

The straight segments are always geodesics, but when the boundary of the convex sets admits coplanar segments, there are infinitely many other geodesics.

The Hausdorff measure associated to the Hilbert metric is in that case a Borel measure $\mu_{\mathcal{C}}$ on $\mathcal{C}$. The metric ball of radius $R$ centred at the point $p$ of the convex domain $\mathcal{C}$ will be denoted by $B_{\mathcal{C}}(p, R)$. The volume entropy of $\left(\mathcal{C}, d_{\mathcal{C}}\right)$ is a constant measuring the exponential volume growth rate of these metric balls and is defined as follows:

$$
\operatorname{Ent}(\mathcal{C}):=\limsup _{R \rightarrow+\infty} \frac{\ln \mu_{\mathcal{C}}\left(B_{\mathcal{C}}(p, R)\right)}{R} .
$$

A Hilbert geometry is said to be divisible when it admits a co-compact subgroup of isometries. In other words it admits a co-compact quotient with respect to the action of that subgroup. In that case, if we consider the orbit of a point of our convex under the action of this group we obtain a discretisation, and as it happens this discretisation is quasi-isometric to our initial geometry. Actually this is true for any Hilbert geometry when we consider a discretisation; see Theorem 1 in Ver09]. However in the general case we don't have a co-compact subgroup of isometries, but one can consider the pseudo group of bounded perturbations of the identity acting on a given discretisation. In the case of a divisible Hilbert geometry and a discretisation induced by the action of the co-compact subgroup, this pseudo group is isomorphic to the latter. That is why a Hilbert geometry is said to be amenable when this pseudo group is, which the author proved to be equivalent to the existence of a Fölner sequence by generalising results of R. Brooks in our setting (see again Ver09).

We are now ready to present our first asnwer. We relate the metric product of two Hilbert geometries with the Hilbert geometry associated to the cartesian product of the two underlying convex sets in the following way:

Theorem 1 (Main proposition). The Hilbert geometry of a product of open convex sets is bi-lipschitz equivalent to the direct metric product of the Hilbert geometries of those convex sets.

The proof of that theorem is surprisingly simple but it allows us to get an impressive range of corollaries, in particular, the existence of a volume decomposition 
inequality (see Corollary 5) which allows one to apply Fubini's theorem. Noticeably with respect to the volume entropy (see also G. Berck, A. Bernig and C. Vernicos BBV10, and M. Crampon [Cra]) and amenability (see C. Vernicos Ver09]) we obtain the following consequences.

Theorem 2 (Main consequences). Consider the two bounded open convex sets $A$ and $B$. Then:

(1) The volume entropy is additive:

$$
\operatorname{Ent}(A \times B)=\operatorname{Ent}(A)+\operatorname{Ent}(B) ;
$$

(2) The product Hilbert geometry $\left(A \times B, d_{A \times B}\right)$ is amenable if and only if both Hilbert geometries $\left(A, d_{A}\right)$ and $\left(B, d_{B}\right)$ are amenable.

Although the product of divisible convex sets need not be divisible itself thanks to the main consequences, one can apply M. Crampon's Cra theorem on volume entropy to get new rigidity results (see Corollaries 7 and 9 in section 3).

Let us conclude with an "opening" remark. Our main theorem shows that a second family of Hilbert geometry seems to play a similar role to the divisible one, those we could call the lip-divisible ones, i.e., whose group of bi-lipschitz bijections admits a discrete subgroup acting co-compactly. Noticeably, following our theorem, this family is closed under product.

\section{Definitions And nOtation}

Finsler stucture. Let $\mathcal{C}$ be a proper open convex set. Let us give an analytical definition of the Finsler metric which gives rise to the Hilbert metric. For any $p \in \mathcal{C}$ and $v \in T_{p} \mathcal{C}=\mathbb{R}^{n}$ with $v \neq 0$, the straight line passing by $p$ and directed by $v$ meets $\partial \mathcal{C}$ at two points $p_{\mathcal{C}}^{+}$and $p_{\mathcal{C}}^{-}$. Then let $t^{+}$and $t^{-}$be two positive numbers such that $p+t^{+} v=p_{\mathcal{C}}^{+}$and $p-t^{-} v=p_{\mathcal{C}}^{-}$. In other words, these numbers correspond to the time necessary to reach the boundary starting at $p$ with the speed $v$ and $-v$. Then we define

$$
F_{\mathcal{C}}(p, v)=\frac{1}{2}\left(\frac{1}{t^{+}}+\frac{1}{t^{-}}\right) \text {and } F_{\mathcal{C}}(p, 0)=0 .
$$

Should $p_{\mathcal{C}}^{+}$or $p_{\mathcal{C}}^{-}$be at infinity, then corresponding ratio will be taken equal to 0 .

Busemann measure. Let us now describe the Hausdorff measure $\mu_{\mathcal{C}}$ on $\mathcal{C}$ in this setting. For historical reasons in the realm of Hilbert geometries, this measure is also known as Busemann measure.

To any $p \in \mathcal{C}$, let $\beta_{\mathcal{C}}(p)=\left\{v \in \mathbb{R}^{n} \mid F_{\mathcal{C}}(p, v)<1\right\}$ be the open unit ball in $T_{p} \mathcal{C}=\mathbb{R}^{n}$ of the norm $F_{\mathcal{C}}(p, \cdot)$ and $\omega_{n}$ the euclidean volume of the open unit ball of the standard euclidean space $\mathbb{R}^{n}$. Consider the (density) function $h_{\mathcal{C}}: \mathcal{C} \longrightarrow \mathbb{R}$ given by $h_{\mathcal{C}}(p)=\omega_{n} / \operatorname{Leb}\left(\beta_{\mathcal{C}}(p)\right)$, where Leb is the canonical Lebesgue measure of $\mathbb{R}^{n}$ equal to 1 on the unit "hypercube". We define $\mu_{\mathcal{C}}$, by

$$
\mu_{\mathcal{C}}(A)=\int_{A} h_{\mathcal{C}}(p) \mathrm{d} \operatorname{Leb}(p)
$$

for any Borel set $A$ of $\mathcal{C}$ (in other words, for any point $p \in \mathcal{C}$ we have $\mathrm{d} \mu_{\mathcal{C}}(p)=$ $\left.h_{\mathcal{C}}(p) \mathrm{dLeb}(p)\right)$. 
Bottom of the spectrum. The bottom of the spectrum of $\mathcal{C}$, denoted by $\lambda_{1}(\mathcal{C})$, and the Sobolev constant $S_{\infty}(\mathcal{C})$ are defined as in a Riemannian manifold of infinite volume, thanks to the Raleigh quotients as follows:

$$
\lambda_{1}(\mathcal{C})=\inf \frac{\int_{\mathcal{C}}\left\|d f_{p}\right\|_{\mathcal{C}}^{* 2} \mathrm{~d} \mu_{\mathcal{C}}(p)}{\int_{\mathcal{C}} f^{2}(p) \mathrm{d} \mu_{\mathcal{C}}(p)}, \quad S_{\infty}(\mathcal{C})=\inf \frac{\int_{\mathcal{C}}\left\|d f_{p}\right\|_{\mathcal{C}}^{*} \mathrm{~d} \mu_{\mathcal{C}}(p)}{\int_{\mathcal{C}}|f|(p) \mathrm{d} \mu_{\mathcal{C}}(p)},
$$

where the infimum is taken over all non-zero lipschitz functions with compact support in $\mathcal{C}$.

Finally, the Cheeger constant of $\mathcal{C}$ is defined by

$$
I_{\infty}(\mathcal{C})=\inf _{U} \frac{\nu_{\mathcal{C}}(\partial U)}{\mu_{\mathcal{C}}(U)}
$$

where $U$ is an open set in $\mathcal{C}$ whose closure is compact and whose boundary is an $n-1$ dimensional submanifold, and $\nu_{\mathcal{C}}$ is the Hausdorff measure associated to the restriction of the Finsler norm $F_{\mathcal{C}}$ to hypersurfaces.

Thanks to CV06 we know that there is a constant $c$ such that

$$
\frac{1}{c} \cdot S_{\infty}(\mathcal{C}) \leq I_{\infty}(\mathcal{C}) \leq c \cdot S_{\infty}(\mathcal{C})
$$

Let us finish by adapting the statement of the main theorem from our paper related to amenability [Ver09] for our present purpose.

Theorem 3. Let $\mathcal{C}$ be a convex domain in $\mathbb{R}^{n}$. The following are equivalent:

(1) $\left(\mathcal{C}, d_{\mathcal{C}}\right)$ is amenable;

(2) $S_{\infty}(\mathcal{C})=0$;

(3) $\lambda_{1}(\mathcal{C})=0$.

\section{The DECOMPosition LEMMA}

Theorem 4. Consider the family of convex domains $A_{i} \in \mathbb{R}^{n_{i}}$, for $i=1, \ldots, k$ and $n_{i} \in \mathbb{N}^{*}$. Then for any point $p=\left(p_{1}, \ldots, p_{k}\right)$ of the convex domain $A_{1} \times \cdots \times A_{k}$ and any vector $v=\left(v_{1}, \ldots, v_{k}\right) \in \mathbb{R}^{n_{1}} \times \cdots \times \mathbb{R}^{n_{k}}$ one has

$$
\max _{1 \leq i \leq k} F_{A_{i}}\left(p_{i}, v_{i}\right) \leq F_{A_{1} \times \cdots \times A_{n}}(p, v) \leq \sum_{i=1}^{k} F_{A_{i}}\left(p_{i}, v_{i}\right) .
$$

Therefore, the identity restricted to $A_{1} \times \cdots \times A_{k}$ is a bi-lipschitz map between $\left(A_{1} \times \cdots \times A_{k}, d_{A_{1} \times \cdots \times A_{k}}\right)$ and the direct product of the metric spaces $\left(A_{i}, d_{A_{i}}\right)$ for $i=1, \ldots, k$.

Proof. Consider a point $p=\left(p_{1}, \ldots, p_{k}\right)$ of the convex domain $A_{1} \times \cdots \times A_{k}$ and a vector $v=\left(v_{1}, \ldots, v_{k}\right) \in \mathbb{R}^{n_{1}} \times \cdots \times \mathbb{R}^{n_{k}}$. If the two positive numbers $t^{+}$and $t^{-}$ are such that

$$
p+t^{+} v \in \partial\left(A_{1} \times \cdots \times A_{k}\right) \quad \text { and } \quad p-t^{-} v \in \partial\left(A_{1} \times \cdots \times A_{k}\right),
$$

then $F_{A_{1} \times \cdots \times A_{k}}(p, v)=\frac{1}{2}\left(\frac{1}{t^{+}}+\frac{1}{t^{-}}\right)$. This implies that for some $i, j \in\{1, \ldots, k\}$, $p_{i}+t^{+} v_{i} \in \partial A_{i}$ and $p_{j}-t^{-} v_{j} \in \partial A_{j}$.

Hence, should we define for each integer $i \in\{1, \ldots, k\}$ the positive numbers $t_{i}^{+}$ and $t_{i}^{-}$by asking that

$$
p_{i}+t_{i}^{+} v_{i} \in \partial A_{i} \quad \text { and } \quad p_{i}-t_{i}^{-} v_{i} \in \partial A_{i},
$$


we would then obtain $t^{+}=\min \left\{t_{1}^{+}, \ldots, t_{k}^{+}\right\}$and $t^{-}=\min \left\{t_{1}^{-}, \ldots, t_{k}^{-}\right\}$, which would imply that

$$
F_{A_{1} \times \cdots \times A_{k}}(p, v)=\frac{1}{2}\left(\max \left\{1 / t_{1}^{+}, \ldots, 1 / t_{k}^{+}\right\}+\max \left\{1 / t_{1}^{-}, \ldots, 1 / t_{k}^{-}\right\}\right)
$$

and therefore using the classical comparison between the $l^{1}$ and $l^{\infty}$ norm in $\mathbb{R}^{k}$ we get

$$
\frac{1}{2} \max _{1 \leq i \leq k}\left\{\frac{1}{t_{i}^{+}}+\frac{1}{t_{i}^{-}}\right\} \leq F_{A_{1} \times \cdots \times A_{k}}(p, v) \leq \frac{1}{2}\left(\sum_{i=1}^{k} \frac{1}{t_{i}^{+}}+\sum_{i=1}^{k} \frac{1}{t_{i}^{-}}\right)
$$

which we can rewrite by associativity of the addition in the following form:

$$
\max _{1 \leq i \leq k} \frac{1}{2}\left\{\frac{1}{t_{i}^{+}}+\frac{1}{t_{i}^{-}}\right\} \leq F_{A_{1} \times \cdots \times A_{k}}(p, v) \leq \sum_{i=1}^{k} \frac{1}{2}\left(\frac{1}{t_{i}^{+}}+\frac{1}{t_{i}^{-}}\right) .
$$

Corollary 5 (Volume decomposition inequality). Consider the family of convex domains $A_{i} \in \mathbb{R}^{n_{i}}$, for $i=1, \ldots, k$ and $n_{i} \in \mathbb{N}^{*}$. Then at any point $p=\left(p_{1}, \ldots, p_{k}\right) \in$ $A_{1} \times \cdots \times A_{n}$ we have the following inequality:

$$
\prod_{i=1}^{k} d \mu_{A_{i}}\left(p_{i}\right) \leq d \mu_{A_{1} \times \cdots \times A_{n}}(p) \leq k^{n_{1}+\cdots+n_{k}} \prod_{i=1}^{k} d \mu_{A_{i}}\left(p_{i}\right) .
$$

Proof. Let us denote by Leb the Lebesgue measure on $\mathbb{R}^{n_{1}} \times \cdots \times \mathbb{R}^{n_{k}}$ normalised by 1 on the unit cube and by $\operatorname{Leb}_{i}$ the corresponding one on $R^{n_{i}}$. Then, for any point $p=\left(p_{1}, \ldots, p_{k}\right)$ of the convex domain $\Pi=A_{1} \times \cdots \times A_{k}$, if $\beta_{\Pi}(p)$ corresponds to the unit tangent ball at $p$ and for all $i, \beta_{A_{i}}\left(p_{i}\right)$ to the unit tangent ball at $p_{i}$ for $A_{i}$, then one has

$$
\frac{1}{k^{n_{1}+\cdots+n_{k}}} \prod_{i=1}^{k} \operatorname{Leb}_{i}\left(\beta_{A_{i}}\left(p_{i}\right)\right) \leq \operatorname{Leb}\left(\beta_{\Pi}(p)\right) \leq \prod_{i=1}^{k} \operatorname{Leb}_{i}\left(\beta_{A_{i}}\left(p_{i}\right)\right)
$$

and the corollary follows by definition of the measure.

\section{THE VOLUME ENTROPY OF PRODUCTS}

The general behaviour of the volume entropy is not yet completely understood, and the main conjecture, to prove that it is always less than that of the hyperbolic geometry, is still open in a dimension larger than 3. Therefore the next result and the generalisation it implies validate this conjecture a little bit more. They also simplify and generalise a result obtained in Ver08.

Proposition 6. The volume entropy is subadditive with respect to a product of convex domains. Take a family of convex domains $A_{i} \in \mathbb{R}^{n_{i}}$, for $i=1, \ldots, k$ and $n_{i} \in \mathbb{N}^{*}$. Then one has

$$
\max _{1 \leq i \leq k}\left\{\operatorname{Ent}\left(A_{i}\right)\right\} \leq \operatorname{Ent}\left(A_{1} \times \cdots \times A_{k}\right) \leq \sum_{i=1}^{k} \operatorname{Ent}\left(A_{i}\right) .
$$

If the convex domains are also bounded, then we actually have additivity:

$$
\operatorname{Ent}\left(A_{1} \times \cdots \times A_{k}\right)=\sum_{i=1}^{k} \operatorname{Ent}\left(A_{i}\right) .
$$


Proof. We will do the proof for $k=2$; the general case trivially follows. Let $A$ and $C$ be two convex domains, respectively in $\mathbb{R}^{n}$ and $\mathbb{R}^{m}$, and let $p=\left(p_{A}, p_{C}\right) \in A \times C$.

Thanks to the left hand side inequality of Theorem 4, we obtain for any point $q=\left(q_{A}, q_{c}\right) \in A \times C$ that

$$
d_{A}\left(p_{A}, q_{A}\right) \leq d_{A \times C}(p, q) \quad \text { and } \quad d_{C}\left(p_{C}, q_{C}\right) \leq d_{A \times C}(p, q),
$$

which imply the next inclusion,

$$
B_{A \times C}(p, R) \subset B_{A}\left(p_{A}, R\right) \times B_{C}\left(p_{C}, R\right) .
$$

The right hand side inequality of Theorem 4 yields in turn that for any $\varepsilon>0$, $B_{A}\left(p_{A}, \varepsilon R\right) \times B_{C}\left(p_{C},(1-\varepsilon) R\right)$ is a subset of $B_{A \times C}(p, R)$.

Hence, computing the volumes, using the inequalities of Corollary 5 we obtain that

$$
\begin{aligned}
\mu_{A}\left(B_{A}(\varepsilon R)\right) \times \mu_{C}\left(B_{C}((1-\varepsilon) p, R)\right) & \\
& \leq \mu_{A \times C}\left(B_{A \times C}(p, R)\right) \\
& \leq 2^{n+m} \mu_{A}\left(B_{A}(p, R)\right) \times \mu_{C}\left(B_{C}(p, R)\right) .
\end{aligned}
$$

Taking the logarithm of both inequalities, dividing by $R$ and taking the limit as $R \rightarrow+\infty$, gives the following inequality, for any $\varepsilon>0$ :

$$
\varepsilon \operatorname{Ent}(A)+(1-\varepsilon) \operatorname{Ent}(C) \leq \operatorname{Ent}(A \times C) \leq \operatorname{Ent}(A)+\operatorname{Ent}(C),
$$

which implies (6).

In case both $A$ and $C$ are bounded, we can work as in CV04] with the asymptotic balls $\mathrm{AsB}_{A \times C}(p, R)$, that is, the image of $A \times C$ by the dilation of ratio $\tanh (R)$ centred at $p$. Those asymptotic balls are exactly the product of the asymptotic balls of $A$ and $C$ respectively centred at $p_{A}$ and $p_{C}$. Therefore,

$$
\begin{aligned}
\mu_{A}\left(\operatorname{AsB}_{A}\left(p_{A}, R\right)\right) & \times \mu_{C}\left(\operatorname{AsB}_{C}\left(p_{C}, R\right)\right) \\
& \leq \mu_{A \times C}\left(\operatorname{AsB}_{A \times C}(p, R)\right) \\
& \leq 2^{n+m} \mu_{A}\left(\operatorname{AsB}_{A}\left(p_{A}, R\right)\right) \mu_{C}\left(\operatorname{AsB}_{C}\left(p_{C}, R\right)\right) .
\end{aligned}
$$

This inequality allows us to conclude (using the fact shown in CV04) that there exists some constant $K$ such that

$$
B_{A \times C}(R-1) \subset \operatorname{AsB}_{A \times C}(p, R) \subset B_{A \times C}(R+K) .
$$

The following corollary is a straightforward application of M. Crampon's Cra] rigidity result and the subadditivity of entropy:

Corollary 7. Consider the family of divisible convex domains with $C^{1}$ boundary $A_{i} \in \mathbb{R}^{n_{i}}$, for $i=1, \ldots, k$ and $n_{i} \in \mathbb{N}^{*}$. Then one has

- $\operatorname{Ent}\left(A_{1} \times \cdots \times A_{k}\right) \leq \sum_{i} n_{i}-k$.

- Equality occurs if and only if all $A_{i}$ are ellipsoids.

Let us denote by $\operatorname{conv}(p, \mathcal{S})$ the convex hull of a point $p$ and a set $\mathcal{S}$.

Corollary 8. Let $\mathcal{C}$ be a convex set in $\mathbb{R}^{n} \subset \mathbb{R}^{n+1}$ and let $p$ be a point outside $\mathbb{R}^{n}$ in $\mathbb{R}^{n+1}$. Then $\operatorname{Ent}(\operatorname{conv}(p, \mathcal{C}))=\operatorname{Ent}(\mathcal{C})$. 
Proof. This comes from the fact that $\operatorname{conv}(p, \mathcal{C})$ is projectively equivalent to $\mathcal{C} \times$ ] $0,+\infty[$, and

$$
\max \{\operatorname{Ent} C, \operatorname{Ent}(] 0,+\infty[)\} \leq \operatorname{Ent}(\mathcal{C} \times] 0,+\infty[) \leq \operatorname{Ent}(\mathcal{C})+\operatorname{Ent}(] 0,+\infty[)
$$

by Proposition 6. As ]0, $+\infty$ [ endowed with its Hilbert geometry is isometric to the real line, we easily conclude the proof.

M. Crampon's Cra rigidity result applied to that case therefore implies:

Corollary 9. Let $\mathcal{C}$ be a divisible bounded convex domain with $C^{1}$ boundary in $\mathbb{R}^{n} \subset \mathbb{R}^{n+1}$ and let $p$ be a point outside $\mathbb{R}^{n}$ in $\mathbb{R}^{n+1}$. Then:

- $\operatorname{Ent}(\operatorname{conv}(p, \mathcal{C})) \leq n-1$.

- Equality occurs if and only if $\mathcal{C}$ is an ellipsoid.

\section{Amenability of products}

Theorem 3 states that a Hilbert geometry is amenable if and only if the bottom of its spectrum is null, which is equivalent to the nullity of its Cheeger constant. In this section we show how this property behaves with respect to product.

Proposition 10. Consider the family of convex domains $A_{i} \in \mathbb{R}^{n_{i}}$ for $i=1, \ldots, k$. The following are equivalent:

(i) The Hilbert geometry of $A_{1} \times \cdots \times A_{k}$ is amenable.

(ii) For all $i, A_{i}$ is amenable.

More precisely, with respect to the bottom of the spectrum and the Sobolev constants we have the following inequalities:

$$
\begin{aligned}
\lambda_{1}\left(A_{1} \times \cdots \times A_{k}\right) & \geq k^{-n_{1}-\cdots-n_{k}} \max _{1 \leq i \leq k} \lambda_{1}\left(A_{i}\right), \\
S_{\infty}\left(A_{1} \times \cdots \times A_{k}\right) & \leq k^{n_{1}+\cdots+n_{k}} \sum_{1 \leq i \leq k} S_{\infty}\left(A_{i}\right) .
\end{aligned}
$$

Proof. Let us denote $A_{1} \times \cdots \times A_{k}$ by $\Pi$. Consider a lipschitz function with compact support $f: A_{1} \times \cdots \times A_{k} \rightarrow \mathbb{R}$. Then we have for almost every point in $A_{1} \times \cdots \times A_{k}$ the function $f$ admits a differential $d f$ and for any $i$ we have $\|d f\|_{A_{i}} \leq\|d f\|_{\Pi}$. Therefore, for any $i$ we have

$$
\int_{A_{1} \times \cdots \times A_{k}}\|d f\|_{\Pi}^{2} d \mu_{\Pi} \geq \int_{A_{1} \times \cdots \times A_{k}}\|d f\|_{A_{i}}^{2} d \mu_{\Pi} .
$$

Now, thanks to Corollary [5, we have for any $i$

$$
\int_{\Pi}\|d f\|_{A_{i}}^{2} d \mu_{\Pi} \geq \int_{\Pi}\|d f\|_{A_{i}}^{2} d \mu_{A_{1}} \cdots d \mu_{A_{k}},
$$

then by the definition of $\lambda, \geq \lambda_{1}\left(A_{i}\right) \int_{\Pi} f^{2} d \mu_{A_{1}} \cdots d \mu_{A_{k}}$,

and thanks to Corollary [5] $\geq \frac{\lambda\left(A_{i}\right)}{k^{n_{1}+\cdots+n_{k}}} \int_{\Pi} f^{2} d \mu_{\Pi}$,

which implies the inequality (10), and the implication (i) $\Rightarrow$ (ii). 
For the other implication we will use the Cheeger constant and, for better clarity, restrict ourselves to the product of two convex sets. Now let us suppose that $I(A)=$ $I(C)=0$ and let us prove that $I(A \times C)=0$. To do so we will prove the inequality (11). Let us consider two real valued lipschitz functions $f$ and $g$ with compact support respectively in $A$ and $C$. We then define the function $h: A \times C \rightarrow \mathbb{R}$ as follows: for any $p=\left(p_{A}, p_{C}\right) \in A \times C, h(p)=f\left(p_{A}\right) g\left(p_{C}\right)$. We first use the textbook equality

$$
d h=g d f+f d g .
$$

Applying the right hand side inequality of Theorem 4 we obtain

$$
\|d h\|_{A \times C} \leq\|d h\|_{A}+\|d h\|_{C} \leq|g| \cdot\|d f\|_{A}+|f| \cdot\|d g\|_{C} .
$$

The next step consists of integrating over $A \times C$, taking into account the right hand side inequality of (5) to obtain

$$
\begin{aligned}
\int_{A \times C}\|d h\|_{A \times C} d \mu_{A \times C} & \\
\leq 2^{n_{A}+n_{C}} & \left(\int_{C}|g| d \mu_{C} \cdot \int_{A}\|d f\|_{A} d \mu_{A}\right. \\
& \left.\quad+\int_{A}|f| d \mu_{A} \cdot \int_{C}\|d g\|_{C} d \mu_{C}\right) .
\end{aligned}
$$

We finish by dividing by the integral of $|h|$ over $A \times C$ using the right hand side inequality of (5) to finally get

$$
\begin{aligned}
& \frac{\int_{A \times C}\|d h\|_{A \times C} d \mu_{A \times C}}{\int_{A \times C}|h| d \mu_{A \times C}} \\
& \leq 2^{n_{A}+n_{C}}\left(\frac{\int_{A}\|d f\|_{A} d \mu_{A}}{\int_{A}|f| d \mu_{A}}+\frac{\int_{C}\|d g\|_{C} d \mu_{C}}{\int_{C}|g| d \mu_{C}}\right) .
\end{aligned}
$$

This last inequality implies inequality (11), and allows us to conclude, thanks to the main theorem of our paper Ver09.

This proposition sheds some light on the example given by proposition 4.1 in CV07. of a Hilbert geometry which is not hyperbolic in the sense of Gromov, but which has positive bottom of the spectrum, and therefore allows us to get more examples of the same kind. Indeed, it is straightforward that a product of convex set is never strictly convex, which implies that it is never hyperbolic in the sense of Gromov.

\section{Illustrations}

In order to illustrate our main theorem in a simple way, we apply it to two geometries: the $n$-dimensional cube and the $n$-dimensional simplex. We also prove that in a tetrahedron, a cone with apex on an edge has finite volume. This answers a question of L. Marquis.

The next two applications are useful to obtain qualitative information on volumes in the given Hilbert geometries (see for instance proposition 6 in CVV04 and its corollaries 6.1 and 6.2 ). 
Proposition 11. Let $\left.\mathcal{C}^{n}=\right]-1,1\left[^{n}\right.$ be the $n$-dimensional cube. We have the following:

(1) $\mathcal{C}^{n}$ is bi-lipschitz equivalent to $\mathbb{R}^{n}$.

(2) For all $\left.x=\left(x_{1}, \ldots, x_{n}\right) \in\right]-1,1\left[{ }^{n}\right.$, let $\beta_{\mathcal{C}^{n}}(x)$ be the tangent unit ball for $F_{\mathcal{C}^{n}}$ at $x$. Then we have

$$
(2 / n)^{n} \prod_{i=1}^{n}\left(1-x_{i}^{2}\right) \leq \operatorname{Leb}\left(\beta_{\mathcal{C}^{n}}(x)\right) \leq 2^{n} \prod_{i=1}^{n}\left(1-x_{i}^{2}\right) .
$$

Notice that actually there is a better lower bound because one can replace $(2 / n)^{n}$ by $2^{n} /(n !)$, using Theorem 4 instead of its corollary.

Proposition 12. Let $\left.\mathcal{S}^{n}=\right] 0,+\infty\left[^{n}\right.$ be the $n$-dimensional positive cone, whose Hilbert geometry is isometric to the Hilbert geometry of the simplex of $\mathbb{R}^{n}$. We have the following:

(1) $\mathcal{S}^{n}$ is bi-lipschitz equivalent to $\mathbb{R}^{n}$.

(2) $\left.x=\left(x_{1}, \ldots, x_{n}\right) \in\right] 0,+\infty\left[{ }^{n}\right.$. Let $\beta_{\mathcal{S}^{n}}(x)$ be the tangent unit ball for $F_{\mathcal{S}^{n}}$ at $x$. Then we have

$$
(4 / n)^{n} \prod_{i=1}^{n} x_{i} \leq \operatorname{Leb}\left(\beta_{\mathcal{S}^{n}}(x)\right) \leq 4^{n} \prod_{i=1}^{n} x_{i} .
$$

For the same reason, one can also replace $(4 / n)^{n}$ by $4^{n} /(n !)$ in this lower bound. In that case one can actually make a precise computation and obtain, for instance,

$$
\operatorname{Leb}\left(\beta_{\mathcal{S}^{n}}(x)\right)=12 x_{1} \cdot x_{2}
$$

Proposition 13. Let $\mathcal{S}^{4}$ be a tetrahedron in $\mathbb{R}^{3}$ endowed with its Hilbert geometry. Consider a cone which is the closure of a disc inside the tetrahedron with an apex on one of its edges. Then the volume of that cone is finite.

Proof. If the disc and the apex are in the same plane the result is straightforward, thus we can suppose that this is not the case. The finiteness only depends on the finiteness around the summit of the cone. Hence we can focus on a neighborhood around the apex. To do so we can notice that there is a prismatic neighborhood around the summit with respect to the tetrahedron, i.e. an edge is a segment centred at the summit included in the tetrahedron's edge the apex belongs to, and the two faces of the tetrahedron whose intersection defines that edge, contains two of the faces of the prism (see also [Ver14]). Besides the comparison of volumes, the volume around the summit of the cone with respect to the Hilbert geometry of this prism is larger than with respect to the tetrahedron's Hilbert geometry. Therefore it suffices to prove the result for such a prism.

Without loss of generality, we can now suppose that: The prism is given by the intersection of the planes $z+x>0, z-x>0, z<1$, and $-1<y<1$. The edge we are interested in will be the intersection of $z=x=0$ and $-1<y<1$ and the summit of the cone is the origin. The cone $\mathcal{C}$ itself will be given in cylindrical coordinates by $\lambda \rho<z<s<1$, with $\lambda>1$. 
Now, thanks to Corollary 5 , we know that the volume of this cone is bounded from above, from some constant $K$, by

$$
I=K \int_{0}^{s} \int_{0}^{z / \lambda} \int_{0}^{\pi / 2} \frac{\rho d \rho d t d z}{\left(z^{2}-\rho^{2} \cos ^{2} \theta\right)\left(1-\rho^{2} \sin ^{2} \theta\right)(1-z)},
$$

where we took into account the symmetries involved for the integral (noticeably the $\pi / 2)$. Now taking into account that $(1-z) \geq(1-s)$ and $\left(1-\rho^{2} \sin ^{2} \theta\right) \geq\left(1-s^{2} / \lambda^{2}\right)$, we obtain that

$$
I \leq \frac{K}{\left(1-s^{2} / \lambda^{2}\right)(1-s)} \int_{0}^{s} \int_{0}^{z / \lambda} \int_{0}^{\pi / 2} \frac{\rho d \rho d t d z}{z^{2}-\rho^{2} \cos ^{2} \theta} .
$$

Now we compute this last integral

$$
\begin{aligned}
\int_{0}^{s} \int_{0}^{z / \lambda} \int_{0}^{\pi / 2} & \frac{\rho d \rho d t d z}{z^{2}-\rho^{2} \cos ^{2} \theta} \\
& =\int_{0}^{s} \int_{0}^{z / \lambda} \int_{0}^{\pi / 2} \frac{d \rho d t d z}{2 \cos \theta}\left(\frac{1}{z-\rho \cos \theta}-\frac{1}{z+\rho \cos \theta}\right) \\
& =\int_{0}^{s} \int_{0}^{\pi / 2}-\ln \left(1-\frac{\cos ^{2} \theta}{\lambda^{2}}\right) \frac{d \theta}{2 \cos ^{2} \theta} d z \\
& =s \int_{0}^{\pi / 2}-\ln \left(1-\frac{\cos ^{2} \theta}{\lambda^{2}}\right) \frac{d \theta}{2 \cos ^{2} \theta} .
\end{aligned}
$$

The integral (21) being convergent concludes our proof.

\section{REFERENCES}

[Ben06] Yves Benoist, Convexes hyperboliques et quasiisométries (French, with English summary), Geom. Dedicata 122 (2006), 109-134, DOI 10.1007/s10711-006-9066-z. MR2295544 (2007k:20091)

[BBI01] Dmitri Burago, Yuri Burago, and Sergei Ivanov, A course in metric geometry, Graduate Studies in Mathematics, vol. 33, American Mathematical Society, Providence, RI, 2001. MR 1835418 (2002e:53053)

[BBV10] Gautier Berck, Andreas Bernig, and Constantin Vernicos, Volume entropy of Hilbert geometries, Pacific J. Math. 245 (2010), no. 2, 201-225, DOI 10.2140/pjm.2010.245.201. MR2608435 (2011c:51008)

[Ber09] Andreas Bernig, Hilbert geometry of polytopes, Arch. Math. (Basel) 92 (2009), no. 4, 314-324, DOI 10.1007/s00013-009-3142-1. MR2501287(2010c:53106)

[CV06] Bruno Colbois and Constantin Vernicos, Bas du spectre et delta-hyperbolicité en géométrie de Hilbert plane (French, with English and French summaries), Bull. Soc. Math. France 134 (2006), no. 3, 357-381. MR2245997(2007g:53080)

[CV07] Bruno Colbois and Constantin Vernicos, Les géométries de Hilbert sont à géométrie locale bornée (French, with English and French summaries), Ann. Inst. Fourier (Grenoble) 57 (2007), no. 4, 1359-1375. MR2339335(2008k:53160)

[CV11] Bruno Colbois, Constantin Vernicos, and Patrick Verovic, Hilbert geometry for convex polygonal domains, J. Geom. 100 (2011), no. 1-2, 37-64, DOI 10.1007/s00022-011-00662. MR2845276 (2012h:51015)

[CVV04] B. Colbois, C. Vernicos, and P. Verovic, L'aire des triangles idéaux en géométrie de Hilbert (French, with French summary), Enseign. Math. (2) 50 (2004), no. 3-4, 203237. MR2116715 (2005k:53125)

[CV04] Bruno Colbois and Patrick Verovic, Hilbert geometry for strictly convex domains, Geom. Dedicata 105 (2004), 29-42, DOI 10.1023/B:GEOM.0000024687.23372.b0. MR 2057242 (2005e:53111) 
[CV] Bruno Colbois and Patrick Verovic, Hilbert domains that admit a quasi-isometric embedding into Euclidean space, Adv. Geom. 11 (2011), no. 3, 465-470, DOI 10.1515/ADVGEOM.2011.016. MR.2817589 (2012g:53148)

[Cra] Mickaël Crampon, Entropies of strictly convex projective manifolds, J. Mod. Dyn. 3 (2009), no. 4, 511-547, DOI 10.3934/jmd.2009.3.511. MR2587084 (2011g:37079)

[dlH93] P. de la Harpe, On Hilbert's metric for simplices, Geometric group theory, Vol. 1 (Sussex, 1991), 97-119. Cambridge Univ. Press, 1993.

[Hil71] D. Hilbert, Les fondements de la Géométrie, édition critique préparée par P. Rossier. Dunod, 1971.

[SM00] É. Socié-Méthou, Comportement asymptotiques et rigidités en géométries de Hilbert, thèse de doctorat de l'université de Strasbourg, 2000. http://www-irma.ustrasbg.fr/irma/publications/2000/00044.ps.gz.

[SM02] Edith Socié-Méthou, Caractérisation des ellipsö̈des par leurs groupes d'automorphismes (French, with English and French summaries), Ann. Sci. École Norm. Sup. (4) 35 (2002), no. 4, 537-548, DOI 10.1016/S0012-9593(02)01103-5. MR1981171(2004j:53055)

[Ver09] Constantin Vernicos, Spectral radius and amenability in Hilbert geometries, Houston J. Math. 35 (2009), no. 4, 1143-1169. MR2577148(2011a:53149)

[Ver08] Constantin Vernicos, Sur l'entropie volumique des géométries de Hilbert, Sém. Th. Spe. et Geo. de Grenoble, 26:155-176, 2008.

[Ver14] Constantin Vernicos, Lipschitz Characterisation of Polytopal Hilbert Geometries. to appear in Osaka J. of Math., preprint 2008.

Institut de Mathématique et de Modélisation de MontPellier, Université Montpellier 2, Case Courrier 051, Place Eugène Bataillon, F-34395 Montpellier Cedex, France

E-mail address: Constantin.Vernicos@um2.fr 